\title{
Persee
}

Mr Dominique Damamme

\section{Histoire et désir d'histoire}

In: Pôle Sud, N¹ - 1994. pp. 61-77.

Citer ce document / Cite this document :

Damamme Dominique. Histoire et désir d'histoire. In: Pôle Sud, N1 - 1994. pp. 61-77.

doi : 10.3406/pole.1994.1326

http://www.persee.fr/web/revues/home/prescript/article/pole_1262-1676_1994_num_1_1_1326 


\section{Histoire et désir d'histoire}

\section{Damamme}

"Naturellement, écrivait F. Kafka, tout le monde peut se dire "grand" s'il lui plaît, mais c'est l'usage judiciaire qui fait autorité". Sauf à introduire une hiérarchie et à participer aux luttes symboliques pour l'imposition d'une définition du bien et du grand, sans doute faut-il, au bout du compte, laisser l'histoire ${ }^{2}$ (ou les sondages ${ }^{3}$ ) trancher le débat. Que le "renom" et la mémoire politique supposent des opérations d'appropriation du mort par le vift, qu'elles tiennent à un tout travail symbolique objectivé dans des institutions, à des pratiques d'inculcation, notamment scolaires et à des rites de célébration, ces affirmations veulent simplement rappeler que les croyances présentes font la grandeur passée, que les morts ont d'abord le pouvoir que leur conferent les luttes des vivants et l'identité que leur prêtent leurs héritiers ou que leur accordent les interprètes de leur mémoire, ce qui ne va jamais sans transformation, altération, voire trahison ${ }^{5}$. Au travers de ces stratégies, ou de ces politiques mémorielles, dont les hommes politiques sont l'objet et l'instrument se redessinent continûment leurs traits et leurs figures posthumes different encore des images multiples et contradictoires que déjà ils endossaient de leur vivant (Ferry famine, Ferry-Tonkin...).

À l'origine de ce travail, on avouera un étonnement, étonnement devant le consen- sus qui, à la mort de Pierre Mendès France en 1982, s'institua autour de sa personne (et qui perdure aujourd'hui). A la vérité, les célébrations du culte avaient commencé avant sa mort : on se souvient de l'accolade entre Francois Mitterrand et Pierre Mendès France lors de la cérémonie d'investiture de 1981 et des paroles alors prononcées : "Sans vous la gauche ne serait pas ce qu'elle est ou elle ne saurait pas ce qu'elle doit être". Ce sentiment d'étonnement, beaucoup, alors, l'éprouvèrent. En 1984, J. P Rioux, dans un article d'humeur sur les cérémonies d'adieux à l'ancien président du Conseil, soulignait déjà que "trop d'hommages défigure".

Après avoir analysé les conditions de production de la légitimité mendésienne, on s'interrogera plus généralement sur les typifications et les constructions identitaires en politique ${ }^{7}$, avant d'esquisser quelques propositions sur les rapports entre identité et stratégie politique. A cet égard, l'enfermement de Pierre Mendès France dans un personnage de "sage" ou de "juste", ressource politique essentielle mais aussi entrave à l'action politique, semble lui avoir interdit tout écart par rapport aux règles normatives qu'il était venu à incarner ${ }^{8}$. 


\section{Pôle Sud}

\section{Consécration}

De tout temps, des hommes, que l'histoire appelle "grands", se sont imposés à l'identification collective de groupes confrontés à des expériences décisives, domination, guerre, famine, épuration, exil. La mémoire collective sacralise les serviteurs d'une "grande" cause, fondateurs de religion, instituteurs de cité, instaurateurs ou restaurateurs de nation, qui, au terme d'un long combat, ont réussi, tels le Prince de Machiavel ou le législateur de J. J Rousseau, à construire une communauté ou à reconstruire une réalité matériellement et symboliquement défaite.

L'immortalité s'attache à ces entreprises de longue course. A ces figures d'ingénieur social et à la représentation presque bourgeoise d'une raison au travail s'oppose l'image guerrière et aristocratique du héros. Le geste héroöque 'est unique, décisoire, souverain; il se définit par une relation à un sens et à un regard collectif. La situation héroïque se déploie sur une scène. Devant un public, le héros exprime les valeurs du groupe par le mépris de sa propre vie ${ }^{10}$, lui qui "préfere l'immortelle renommée aux choses mortelles", lui dont la mort assure l'immortalité dans la mémoire des hommes ${ }^{11}$. Topos rhétorique ${ }^{12}$, le parallèle entre le grand homme et le héros est classique. Pour Voltaire, "un roi-soldat est appelé héros. Un monarque-législateur, fondateur et guerrier est le véritable grand homme; et le grand homme est au dessus du héros"'13. Peut être en est-il encore ainsi au $\mathrm{XX}^{\mathrm{c}}$ siècle. Crédit immense et pourtant fragile que celui du grand homme politique car souvent sa légitimité surgit dans des temps de crise, au plus profond de l'évanouissement d'une souveraineté. Son autorité, si l'on se reporte aux exemples de ce siècle, supporte mal, au moins dans les démocraties parlementaires, le rétablissement de l'ordre institutionnel, très prosaïquement la réapparition des routines institutionnelles et politiques, surtout si lui manquent, comme au prophète désarmé du Principe, les armes de sa politique, en l'occurrence la faveur incontestable du suffrage et de solides ressources partisanes ${ }^{14}$.

L'immortalisation des fondateurs et des héros apparaît comme la forme extrême de l'idéalisation du pouvoir. Si l'on suit $M$. Weber, le charisme du démagogue, par l'adhésion d'une "communauté émotionnelle" ${ }^{15}$, ou les qualités du "vrai" homme politique - la passion, le sens des responsabilités, le coup d'œil "froid" - suffirait amplement pour vivre dans la mémoire collective, l'analyse du sociologue allemand, peu sensible à l'enchantement démocratique, opérant ici, sous couvert de définition, une évidente valorisation d'un certain modèle d'homme politique.

Analysant l'autorité politique de Pierre Mendès France, René Rémond, il y a longtemps déjà, insistait sur le paradoxe $d$ ' "une réputation indiscutée et le peu de circonstances qu'il eût à la justifier". Ce décalage entre la durée d'occupation du pouvoir et le crédit politique a donné naissance à l'idée d'un Mendès "mythe vierge" en lequel on a pu voir avec quelque raison "le fin mot de l'histoire"16. On peut penser en effet que la brièveté de l'expérience gouvernementale n'a pas laissé à la désillusion et au désenchantement le temps de s'installer 


\section{Histoire et désir d'histoire}

et que l'innocence politique de Mendès France a favorisé une multiplication d'investissements, les mendésismes précisément, adonnés, chacun avec sa singularité, à faire des diverses "faces" de l'homme politique ou des diverses séquences de sa carrière un objet d'exemplarité. Mais pour que ces constructions sociales (contemporaines ou posthumes) revêtent un commencement de vraisemblance et de consistance, encore faut-il un matériau. Un héritage ne part pas de rien, ne se bâtit pas sur du sable, et l'économie des légitimations suppose des faits, une "réalité". En d'autres termes, tout, ici comme ailleurs, n'est pas que construction.

Cette réalité, comment pourrait-on la méconnaître et en même temps comment l'histoire ou la sociologie peut-elle en rendre compte? Faute d'une analyse structurelle, la biographie prendra la forme d'un "Miroir" du prince, d'un hommage, courra le danger de se réduire en un bilan des attributs socialement validés, en une comptabilité des sens rétrospectivement construits de la carrière de Mendès France ${ }^{17}$.

Précisément, rares apparaissent les textes qui échappent à la tentation de l'histoire héroïque ou sainte ou tiennent jusqu'au bout un parti pris réaliste ${ }^{18}$. Tout se passe comme si l'objet "Mendès" était empreint de sacralité, comme si le respect dû à un personnage éminent (qu'il s'agisse ici de P. Mendès France ou, en d'autres temps, d'autres lieux et pour d'autres causes, de Charles de Gaulle) rendait problématique l'objectivation scientifique. Bon nombre de travaux "savants" contribuent ainsi au mouvement général de grandissement de l'homme politique Mendès France, à son "renon"19, et participent, en instituant sa personne en figure éponyme de la République, à la célébration de valeurs politiques et morales dont sa vie porte ou porterait témoignage. On peut ainsi lire - on "apprend" - que l'échec de Pierre Mendès France a tenu à une "exigence morale", à sa "passion d'avoir raison", au fait qu'il ne se sentait pas à l'aise dans une organisation politique. Tout ceci n'est sans doute pas faux et peut-être pas aussi idéaliste qu'il semble, mais pourquoi s'arrêter là, et pourquoi ne pas tenter l'interprétation des dispositions, ressources, lignes d'action et actes d'un homme qui a été aussi et peut être d'abord un "grand" professionnel de la politique. Faute de quoi, l'étude biographique produira un effet de légitimation en raison du modèle implicité de "cité civique" ${ }^{20}$, fait de valorisation du politique et de défense de la démocratie ${ }^{21}$, qui la sous-tend. Mais le grandissement de la personne peut également procéder de l'opération de décontextualisation des interdépendances que génère souvent le travail de singularisation d'un sujet singulier ; l'occultation du réseau général des interactions politiques suffit à contribuer au processus général de légitimation, surtout lorsqu'il s'agit d'un personnage politique hors du commun. Bref, si Mendès France apparaît dans ces récits hors espace politique, extrait du jeu politique et comme abstrait du monde social, c'est d'abord que l'homme a rejoint un idéal et s'est transformé en norme : tout alors fait preuve ou fait sens dans une prosopopée de l'exemplarité. Mais c'est aussi et plus fondamentalement en raison de l'oubli de l'impératif d'analyser relationnellement l'activité politique. 


\section{Pôle Sud}

La désignation comme secrétaire d'État dans le second gouvernement Blum de 1937 fonctionne comme un premier signe d'excellence et d'élection; s'y ajoute sa conduite pendant la guerre - le procès de Clermont-Ferrand, l'évasion, l'engagement dans la R.A.F -, associée à sa nomination par le général de Gaulle à la tête du ministère de l'Économie nationale et à sa démission qui devient symbole de rigueur. Par la suite sont relevées ses prises de position en matière de politique économique et de politique internationale à propos du conflit indochinois, et plus encore les "principes", la science, la morale, dont se réclame la "République Moderne". Dans cette histoire ex post, la période d'exercice du pouvoir est décisive : y contribuent les décisions prises mais aussi le style de gestion de l'agenda gouvernemental que particularisent la dramatisation de l'auto-engagement, la langue, qualifiée de "parler-vrai" - "j'ai la tâche, certains diront ingrate, d'avoir à parler de sacrifices ${ }^{22}$-, et la gestion très attentive de la communication publique, en particulier par le canal de L'Express, "journal pour Mendès France"23, du Monde ${ }^{24}$ et des causeries radiophoniques. Qui aujourd'hui se souvient de la phrase d'Ernest Renan, rétrospectivement cruelle, qui accompagnait la photographie de Mendès France sur la couverture du premier numéro de L'Express : "Le grand politique est celui qui réussit et non celui qui aurait dû réussir" (24 juillet 1953). Si lors des Accords de Genève Mendès joue un rôle que l'intéressé lui-même a comparé à celui d'un syndic de faillite, la paix en Indochine et les Accords de Paris accréditent définitivement sa réputation d'homme d'État. Tout ceci - qui n'est pas rien - fonde une autorité politique considérable et déjà une référence dans une période où les partis politiques de gauche paient la politique algérienne de la IV République et l'arrivée au pouvoir du général de Gaulle.

Aussi validantes qu'aient été ces ressources, leur poids a varié en fonction du contexte politique, s'affaiblissant notablement après 1958, avec l'arrivée au pouvoir du Général de Gaulle. Loin, comme on le pense souvent, d'être pourvu d'une légitimité pérenne, Mendès France a dû batailler pour entretenir ou rétablir son droit à la parole. Alors que sa position politique est fortement entamée par le processus de recomposition de la Gauche à partir de 1962, il parvient cependant à revenir sur le devant de la scène politique entre 1967 et 1969 puis à s'y maintenir sous la double forme d'une carrière "morale" et d'expert. Dans sa dernière période d'activité politique, son autorité sera mise en péril au moins à deux reprises : après sa participation au meeting de Charléty (27 mai 1968) et lors de son association, "hypocrite" aux dires mêmes de certains fidèles, avec G. Defferre en $1969^{25}$, situation-limite où se déchiffre l'impossibilité d'échapper sans reniement et sans dommage au personnage qu'il est devenu, celui d'un intellectuel, d'une "conscience", d'un sage, c'est à dire aussi, en un sens, d'un "mort politique". C'est d'ailleurs dès sa mise à l'écart que Pierre Mendès France commence à constituer un stock de signes et d'exemples dans lequel certains puisent un modèle à invoquer ou un héritage à revendiquer.

Mais ces indications ne livrent encore 


\section{Histoire et désir d'histoire}

qu'une simple description du crédit politique de P. Mendès France.

Son autorité se nourrit d'abord du temps. On rappellera qu'il est devenu parlementaire très jeune, en 1932, à 27 ans, et que "Jeune-Turc" du Parti radical, il accède à 31 ans à un poste ministériel. En 1953, Pierre Mendès France est encore un "jeune" en politique, ce que relève Vincent Auriol lors de l'entretien qui précède sa désignation à la présidence du Conseil : "Je voudrais sortir des sentiers battus. Homme jeune s'appuyant sur des jeunes... vous pouvez faire une nouvelle majorité avec un programme hardi..." A quoi Mendès répond : "Je suis détesté de ce qu'on appelle les "grands" hommes politiques de ce pays. Ça m'est égal. J'ai par contre de grands encouragements de jeunes et c'est ce qui compte. ${ }^{26}$ Au total, il va accumuler plus de cinquante années de vie publique. Or, la longévité constitue une condition essentielle de la reconnaissance par les pairs et par l'opinion. Elle lui a permis de surmonter l'échec, de s'approprier des thèmes (dans le cas de Mendès France : la paix, la décolonisation, la modernisation économique, la rigueur, la gauche, le socialisme) qui ont cristallisé son identité politique, ou encore de renouveler ses équipes de fidèles - phénomène décrit par les termes de "nébuleuse" ou de "diaspora" mendésiste $e^{27}$.

La durée a autorisé également l'agrégation de générations militantes. Sous ce rapport, son adhésion au PSU, parti jeune et de jeunes, apparaît cruciale, non seulement parce qu'il offre un point de saillance à la jeunesse - à la jeunesse des écoles, à la jeunesse politisée engagée dans la lutte contre la guerre d'Algérie -, mais aussi parce que, rééditant symboliquement le geste de 1954, il lie deux générations et deux populations militantes, (dé) montrant ainsi son appartenance au camp de la gauche et la continuité d'une même ligne politique en faveur de la paix. Il réitérera d'ailleurs cet "investissement" sur la jeunesse en 1968 avec sa participation au meeting du stade Charléty, et quoiqu'on ait souvent interprété sa présence comme un faux-pas, il n'est pas sûr qu'elle ait été totalement improductive puisqu'elle confortait son droit à représenter le présent et l'avenir. En définitive, on tient, avec cette visée en direction de la "jeunesse", une des bases les plus fermes et la plus durable de la légitimité mendésienne et on comprend pourquoi il a pu à la fois rester "jeune" et devenir immortel : cette identification lui a procuré, en même temps que des fidèles et des célébrants, des strates successives de publics prédisposés, du fait de leur socialisation politique, à l'entendre. C'est cette superposition de plusieurs strates (trois, au moins) de publics jeunes, à fort capital intellectuel, appelés par la suite à exercer des positions de pouvoir et de savoir, qui a permis à P. Mendès France de devancer le temps, en partie de l'abolir.

La mort de P. Mendès France a imposé au personnel politique une obligation de déférence, conférant aux déclarations l'apparence et la force des sentiments et des révérences partagées. Il s'agit là d'une forme classique de l'expression obligatoire des sentiments qu'a étudié Marcel Mauss ${ }^{28}$. Quelques citations, en vrac : F Mitterrand, "La France a perdu un des plus grands de ses fils" ; J. Chirac, "Mendès France, grand 


\section{Pôle Sud}

résistant, fut à la Libération, le collaborateur du général de Gaulle, qui rendit hommage à son exceptionnelle valeur. Au delà des divergences politiques, je m'incline avec respect devant ce grand serviteur de la France" ; A. Lajoinie, "C'est un homme d'État important qui vient de disparaître" ; M. Rocard, "Il guide notre route pour aujourd'hui et pour demain" ${ }^{29}$. L'unanimité surgit d'un double mécanisme : d'une part, le silence ou l'adieu pacifié des anciens adversaires, de l'autre, l'hommage des amis et d'abord des héritiers présomptifs. On retiendra, entre de nombreux possibles, cette parole de M. Rocard : une filiation "qui honore et rend humble"; ou cet extrait d'un entretien entre L. Fabius, photographié au pied de la statue de P. Mendès France, et le danseur J. C. Galotta : Pierre Mendès France "s'attachait toujours à donner du sens à son action politique. Donner du sens, c'est-à-dire à la fois une direction et une interprétation. J'ai beaucoup réfléchi à la fameuse méthode Mendès quand Mitterrand m'a nommé à Matignon. La quintessence de sa conception de la politique, c'était de rendre les citoyens co-intelligents de la situation, des projets des décisions. Une haute conception de la politique ; la seule qui vaille. ${ }^{{ }^{30}} \mathrm{~A}$ sa mort, Mendès France apparaît ainsi comme l'incarnation du métier politique et comme la démonstration définitive de l'excellence du régime démocratique. Ce que sa mort a autorisé, ce fut, moment rare, une magnification collective et collégiale de l'activité politique et du régime représentatif. Elle fut célébrée comme il se devait et on comprend qu'elle soit l'objet de commémorations répétées.
L'exemplarité mendésienne s'avère pourtant à double tranchant, idéal de l'homme politique, mais aussi instrument de critique du système représentatif. On rappellera ce jugement désabusé de G. Suffert : "l'avenir dure longtemps. Tout peut un jour arriver, même ceci, qu'un acte conforme à l'honneur et à l'honnêteté apparaisse, enfin de compte, comme un bon placement" ${ }^{31}$; ou cette leçon que croit pouvoir retenir $M$. Rocard : "Dire la vérité, annoncer son calendrier et ses choix, tenir ses engagements, parler la même langue à ses partisans et à ses adversaires, c'est d'abord marquer pour autrui une reconnaissance qui est gage non seulement de démocratie mais aussi d'efficacité... que tout discours n'est pas nécessairement voué à devenir mensonge, que toute promesse ne sera pas forcément trahie,... reste une idée profondément neuve et moderne." ${ }^{32}$ Positive, mais pour celui-là qui se proclame disciple, la référence à Mendès France renferme une puissance corrosive pour tout pouvoir en place. On devine que ce ne fut pas (que ce n'est pas) le moindre de ses charmes.

A l'annonce de sa mort, quotidiens et hebdomadaires consacrent un dossier spécial au "dernier des justes", comme titrent ensemble Le Nouvel Observateur et Les Nouvelles Littéraires. Obligation de déférence là aussi, certes diversement observée selon les organes de presse, et à l'intérieur d'un même journal, spontanément acquittée lorsque Mendès France appartient à la "famille", qu'il est un "ami" dans la langue indigène du "Nouvel. Observateur" ${ }^{33}$. Pour tous, pour la presse de province comme pour la presse de droite, cette disparition constitue un événement politique majeur. 


\section{Histoire et désir d'histoire}

Et en tant que tel, il comporte une exigence d'élévation, à la hauteur de l'homme, à la dimension de l'Histoire : propos ou prétexte à des commentaires en forme de leçons que le "système", le "régime" ou, plus simplement, le "gouvernement" doit tirer. A nouveau, s'affirme l'usage ambivalent de la référence mendésienne, instrument de légitimation et d'évaluation de la politique et des hommes qui la font.

Cette promotion au rang d'impératif catégorique de Mendès France, commune au champ journalistique et au champ politique, se retrouve dans le champ intellectue $^{34}$. Un des rapporteurs du colloque de l'Institut d'Histoire du Temps Présent notait justement que la représentation mendésienne apparaît le produit rétrospectif de la légitimité acquise par les jeunes intellectuels des années cinquante ou soixante. "L'auréole intellectuelle dont ils (les fidèles) parent le courant mendésiste est davantage rétroactive que contemporaine de ce courant... plus généralement, ce sont les intellectuels qui (...) sont devenus les gardiens de la flamme du mendésisme" ${ }^{35}$. L'autorité de Mendès France a ainsi cru au fur et à mesure de la promotion sociale de ses jeunes fidèles. En fait, pour tout un ensemble de raisons morphologiques et idéologiques, le rapport des intellectuels à Mendès France s'est modifié entre les années cinquante et les années soixante-dix dans le sens d'un accroissement de légitimité. Sur la base d'une distinction grossière mais commode entre trois types-idéaux d'intellectuels et, parallèlement, trois formes de posture, idéologique, morale et réaliste ${ }^{36}$, on constate d'abord l'opposition des "intellectuels orga- niques" du Parti communiste à la politique de Mendès (à l'exception de sa politique coloniale). Parmi les "intellectuels à la première personne" ${ }^{37}$, et pour ceux qui s'identifient à la figure de la révolte morale Sartre, évidemment, et l'équipe des Temps Modernes -, la prise de distance est nette : "L'attitude criminelle du gouvernement en face des problèmes nord- africains, son obstination à réarmer l'Allemagne, son programme économique qui dissimule mal l'attentisme sous de prétendues réformes, tout cela finira par ouvrir les yeux aux gens et par révéler la vérité de ce qu'il faut bien appeler la mystification Mendès France" ${ }^{13}$. Mythe donc, mais mythe mystificateur. Aux yeux de ces intellectuels "éthiques" et du segment du champ intellectue ${ }^{39}$ qui se reconnaissent en eux, l'“idée républicaine" avait, semble-t-il, épuisé depuis longtemps son sens. En témoigne le numéro spécial consacré par les Temps Modernes à la gauche en 1955 qui fait figure de contre-feu à l'entreprise mendésienne ${ }^{40}$. Entre l'homme politique et cette fraction du monde intellectuel, les rapports s'infléchiront par la suite sans toutefois $s^{\prime}$ inverser ${ }^{41}$. En fait, Mendès France fut consacré à un autre pôle, universitaire, du champ intellectuel, moins d'ailleurs par les réalistes à la manière aronienne que par le courant réformiste. L'éclat de rire qui secoue les étudiants à l'invite du président du Conseil d'adhérer au Parti radical ${ }^{42}$ sanctionne un personnel politique dévalué, non les valeurs qu'il proclame. Le "plus intellectuel de nos hommes politiques", critiqué par les prophètes, fut reconnu par les universitaires (présents et futurs) ${ }^{43}$ qui ont vu en lui l'un des leurs ${ }^{44}$, un homme politique qui, loin 


\section{Pôle Sud}

du mépris ordinaire de ses semblables pour les "chers professeurs" 45 , recherchait leur compagnie, les consultait, et incarnait la politique comme elle devait être, une activité morale et une pratique théorique : gouverner, c'est prévoir, c'est choisir et c'est donc penser. Le pouvoir d'attraction de Mendès France résidait (et réside encore) dans l'affirmation du double lien entre science et politique - dans une définition intellectuelle de la politique comme science en action et dans une manière intellectuelle de la conduire - et entre morale et politique $^{46}$. Au total, la proximité des habitus a pleinement fonctionné et, à l'évidence, l'enchantement continue d'opérer ${ }^{47}$.

Le champ politique peut être saisi comme un système de relations concurrentielles pour la conquête de positions de pouvoir et, plus précisément, pour le droit à représenter. Cette lutte touche aussi la production des biens politiques, et, sur ce terrain, plusieurs catégories d'acteurs, en dehors des hommes politiques et des journalistes, entrent en jeu, les intellectuels mais aussi la haute administration. Certains secteurs de la haute fonction publique ont activement appuyé l'action de Mendès France dans une entreprise de conquête des "sommets" de l'État. Ici, deux séries de facteurs ont joué : d'une part, une construction commune de la politique économique - la "modernisation n'a d'autre alternative que la décadence" 48 - et, plus sans doute que le contenu concret des décisions, une même représentation des rapports entre économie et politique ; d'autre part, la carrière administrative de Mendès. En 1946 il devient "executive director" de la BIRD et gouverneur du FMI; en 1947, il est Délégué permanent au Conseil économique et social de l'ONU. C'est à ces titres qu'il donne en 1947 et 1948 un cours à l'ENA sur la politique des investissements. En 1952, il est nommé président de la Commission des comptes de la nation, fonction qu'il exerce sans interruption jusqu'en 1960. Il est peu d'hommes politiques qui, en son temps, peuvent se prévaloir d'un tel espace positionnel, à l'intersection des secteurs politique, administratif, universitaire et journalistique. "Intellectuel organique" des catégories dirigeantes, il fait fonction de "passeur" à une époque où les communications entre le politique, l'économique et l'administratif restent rares. "Il était, dira F. Bloch-Lainé, un correspondant de l'autre côté de la ligne de démarca-

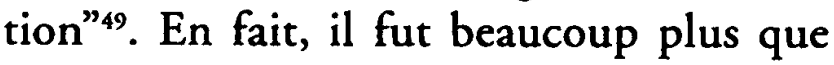
cela si l'on suit l'enquête de F. Fourquet sur les "comptes de la puissance"so, où il apparaît comme un des protecteurs de la comptabilité nationale (après l'épisode Pinay), la caution d'une "avant-garde" administrative et le porte parole ou l'énonciateur d'une vision du monde. On retiendra quatre éléments, d'importance croissante : le surplus de légitimité que Mendès tire de sa position d'expert; l'appui qu'il trouve auprès d'un groupe de "jeunes" hauts fonctionnaires, soudé par une stratégie commune, politique et professionnelle et doté des propriétés des groupes à mémoire au fondement de célébrations continuées ${ }^{51}$; le soutien, de caractère exceptionnel sous la $I^{e}$ République, mais qu'on retrouvera avec le général de Gaulle, de segments significatifs de la haute administration (son gouvernement est composé pour plus de $37 \%$ de hauts fonctionnaires, proportion très supé- 


\section{Histoire et désir d'bistoire}

rieure à la moyenne des formations gouvernementales de la $\mathrm{V}^{\mathrm{c}}$ République $^{52}$ ) qui élargit et conforte les liens établis avec la presse et les universitaires et institue Mendès en position de médiation pour la production et la diffusion des biens politiques ; l'anticipation objective des transformations structurales du champ politique - c'est à dire de la transformation du recrutement politique et des enjeux - qui fait de lui un précurseur, condition d'abolition du temps et de la pérennisation de ses positions. Au delà des incertitudes, souvent exprimées, sur la localisation politique de P. Mendès France, notamment par rapport à l'opposition droite/gauche, puisque son "programme" brouille les lignes de clivage de la IV République, liant des thématiques jusqu'alors disjointes de la république et de l'économie en une synthèse difficilement acceptable dans la problématique politique dominante du temps, ce qui faisait enjeu et était en jeu, c'était une nouvelle définition de la politique légitime et une autre manière de faire légitimement de la politique, toutes choses qui sont passées dans l'histoire sous le nom de modernité ou de modernisation politique.

On est sans doute mieux en mesure, à présent, de répondre à l'interrogation de départ : la force du mendésisme et la diversité des entreprises de célébration trouvent leur explication dans la multipositionnalité de l'intéressés ${ }^{53}$, dans la possession de ressources multiples, dans l'intensité des transactions avec différents secteurs - universitaire, administratif, politique -, et dans la mobilisation d'un "capital" politique assurément hors du commun par des entrepreneurs politiques, des équipes administra- tives, des réseaux intellectuels, ou même des strates générationnelles à "mémoire" intéressés à des titres extrêmement divers par l'usage de la marque et plus tard de la référence.

\section{L'attrait de l'acteur}

Genre traditionnel des études littéraires et historiques, la biographie a souffert en sociologie et en histoire sociale de la dévalorisation attachée à la connaissance individuelle $^{54}$. Si la démonétisation de la conception marxiste du social, si les problématiques du sujet, et dans le public, le refus de la modernité romanesque et l'adhésion à la représentation classique de l'individuss, expliquent son succès actuel, l'approche biographique dans les sciences sociales demeure largement suspecte de biais, et tout particulièrement d'illusion généalogique et essentialiste ${ }^{56}$.

Travailler à partir du discours indigène des acteurs du champ politique - autobiographies, biographies, témoignages - pose d'autres problèmes tout aussi considérables : "l'illusion biographique", la logique de rationalisation du passé, le travail de légitimation qui portent ces récits, leur idéalisme historique, et en dehors même du versant hagiographique ${ }^{57}$, le danger de la tentation héroïque et de l'esthétisation de la politique.

Mais une fois prises au sérieux ces critiques, et d'autres possibles, le débat sur "la" méthode biographique parait quelque peu irréel en raison de la diversité des 


\section{Pôle Sud}

perspectives dans lesquelles s'inscrivent les usages de la biographie ${ }^{58}$. Par ailleurs, aussi problématique que se révèle l'instrument, il serait absurde d'en conclure à l'invalidation ou à l'oblitération du matériau qu'il procure. D'ailleurs, si l'on en juge par l'attention souvent nominale qu'accordent les analyses du personnel ou de l'engagement politique aux dispositions ${ }^{59}$, aux formes d'idéal du moi ${ }^{60}$ et aux identités politiques, le danger majeur réside moins dans le traitement que ces éléments sont susceptibles d'offrir que dans leur oubli ${ }^{61}$.

Soit donc la question : comment rendre sociologiquement compte de certaines attitudes, notamment du rigorisme et du caractère doctrinaire qu'on se plaît à reconnaître chez Pierre Mendès France, et qui apparaissent au principe de son élévation au rang d'homme d'Etat, presque de saint républicain.

Si l'on s'attache à ce que l'on nommera la production publique du Soi, la face publique ou les identités politiques, phénomènes pour partie "externes" aux individus, c'est un fait connu que tout homme public se trouve progressivement enfermé dans des objectivations dont il est à la fois le producteur et le produit. En un sens, l'homme public est aussi un auteur en quête d'un personnage : il se sert des catégories politiques (démocrate, libéral...) et de typifications comme celles de militant, de parlementaire, voire d'homme d'État, qui contribuent à rendre logiquement et chronologiquement cohérentes ses actions, à ses yeux et aux yeux des autres. "Même le caractère quelque peu mythique dont on décore mon personnage contribue à répandre l'idée que des obstacles, par tous autres infranchissables, vont s'aplanir devant moi... me voici, toujours contraint par l'exceptionnel crédit que me fait le peuple français, me voici, obligé autant que jamais d'être ce de Gaulle à qui tout ce qui arrive au-dedans et au-dehors est personnellement imputé, dont chaque mot, et chaque geste, même quand on les lui prête à tort, deviennent partout des sujets de discussion dans tous les sens et qui, nulle part, ne peut disparaître qu'au milieu des clameurs ardentes. Éminente dignité du chef, lourde chaîne du serviteur"62. Ces objectivations s'imposent de l'extérieur et constituent des contraintes d'image. Celles-ci équivalent à des devoirs, la fidélité à soimême, le point d'honneur, l'obligation de ne pas déchoir. Le grand homme est souvent condamné à la reproduction, à l'identité, à être à la hauteur de la définition qu'il s'est donné et qu'il se donne parce que les autres la lui ont donné, en tout cas, à ne pas trop s'écarter du personnage qui constitue sa personne. Ces figures que l'acteur contribue pour partie à façonner, et, pour une autre part, nées de l'amour de ses fidèles, une fois "bien fondées", lui deviennent comme extérieures et pèsent sur son activité. Elles lui collent à la peau, tout comme le succès d'un comédien l'enferme dans un certain type de rôle. Ces constructions dotées d'une réalité sur laquelle l'intéressé n'a qu'une prise relative, peuvent devenir, au "cinéma" comme sur la scène politique, des entraves à l'action. Par exemple, Pierre Mendès France ne peut pas, ni en 1968 ni en 1969, faire personnellement acte de candidature à la présidence d'une République dont il a dénoncé 


\section{Histoire et désir d'histoire}

le caractère autoritaire (mais cela ne gêne aucunement F. Mitterrand) ; Pierre Mendès France ne peut pas en tant qu'autorité morale, se livrer, tout comme un autre, à des actes qui pourraient être interprétés comme des manœuvres "politiciennes" contraires à sa réputation d'intégrité (ainsi passer des alliances "contre-nature" pour emporter la direction d'un parti comme le fait F. Mitterrand à Epinay). Il arrive ainsi que les objectivations emportent des règles normatives qui interdisent le recours aux règles pragmatiques du métier, condamnent un éventuel retour à la politique active et obligent à se satisfaire d'une carrière "morale".

Cependant, à partir du moment où l'analyse s'attache à un individu, il n'est pas possible de laisser de côté les dispositions et attitudes ainsi que les images de soi, effets de la rencontre des dispositions et des expériences mais aussi de l'auto-analyse de l'acteur et de son travail de soi sur soi, travail réflexif qui n'est pas propre à la vie politique mais qui est impliqué par toute vie publique et toute prétention à une vie publique. Il n'est pas anodin, par exemple, que Charles de Gaulle, en 1928, ait consacré la partie centrale du Fil de l'épée à l'homme de caractère. Qui ne connaît la phrase d'exergue, "Etre grand, c'est épouser une grande querelle", et les lignes consacrées au chef et au prestige : "Face à l'événement, c'est à soi-même que recourt l'homme de caractère. Son mouvement est d'imposer à l'action sa marque, de la prendre à son compte, d'en faire son affaire... l'homme de caractère confere à l'action sa noblesse; sans lui morne tâche d'esclave, grâce à lui, jeu divin du héros...
Fait affectif, suggestion, impression produite, sorte de sympathie inspirée aux autres, le prestige dépend, d'abord, d'un don élémentaire, d'une aptitude naturelle qui échappent à l'analyse. Le fait est que certains hommes répandent, pour ainsi dire de naissance, un fluide d'autorité dont on ne peut discerner au juste en quoi il consiste et dont on s'étonne parfois tout en subissant les effets. ${ }^{63^{\prime \prime}}$ C'est bien là un signe tangible d'une "tentation de l'histoire" d'avant l'Histoire qu'il faut prendre en compte et dont il faut rendre compte. Certes, c'est ce type de formation "grandiose" du moi, ce sont ces images ou ces idéalisations glorieuses de soi que l'on décèle ici, anticipations narcissiques de l'avenir, souvent improbables et pourtant advenues, qui, lorsque l'historiographe les rencontre, deviennent le signe d'un destin et contribuent à entretenir la croyance en une sorte de prédiction créatrice ${ }^{64}$ ou la vision idéaliste d'une vie totalement accordée à son projet. "En somme, je ne doutais pas que la France devrait traverser des épreuves gigantesques, que l'intérêt de la vie consistait à lui rendre, un jour, quelque service signalé et que j'en aurai l'occasion" "s. Mais ce n'est pas nécessairement déifier son sujet ou succomber à la tentation héroïque que de s'intéresser aux projections et constructions que véhiculent les "grandes espérances". Tocqueville, dans La démocratie en Amérique, oppose démocratie et héroïsation de la vie. Cette déréliction démocratique, qui tiendrait à la valorisation sociale de l'autonomie et à la passion égalitaire, laisserait ainsi peu de place à la recherche de la gloire comme d'ailleurs à la représentation de l'immortalité. Or, à 


\section{Pôle Sud}

l'inverse, on pourrait soutenir que la finitude propre à une "société des individus" tend à se nier elle-même ${ }^{66}$. Loin d'avoir disparu avec les sociétés aristocratiques, les projections "héroïques" de soi subsistent, dans certains groupes, sous la forme d'un éthos de l'honneur. On pourrait plus simplement penser à la force de certaines identités sociales qui s'expriment dans les valeurs de dévouement, du don de soi, mais aussi de la sainteté ou même du martyre, toutes figures de l'exemplarité, ou évoquer encore le désir de reconnaissance ou la recherche d'un dépassement de la finitude. Comme le déclarait il y a peu un ancien Premier ministre, devant l'horizon de la mort, "la politique, par définition, dépasse le temps de ma propre vie"67.

Certes, ces dispositions et ces images, liées à des conditions d'apprentissage et à des chances d'actualisation, sont inégalement distribuées selon les groupes sociaux. Tout n'est pas écrit d'avance, mais tout n'est pas possible, comme le montre la nouvelle de J. P Sartre L'enfance d'un chef. La solidification ou la désagrégation de ces cristallisations tient à l'ajustement des espérances et des expériences plus sans doute qu'à une connaissance intellectuelle des chances de réussite. Le démenti de la pratique provoque un phénomène d'accommodation, à moins qu'un mécanisme de dissonance cognitive ${ }^{68}$ ne vienne défendre aspirations et prétentions ${ }^{69}$. A l'inverse, le succès conforte la certitude de soi. Il confirme des droits : autorité, sentiment de supériorité, sens de sa dignité, de sa grandeur parfois." Et moi aussi, je suis peintre"70. Mais, quelque soient les conditions d'apparition, quelque soit le degré de résistance de ces représentations mentales et de ces attentes, si ce qui est en jeu, c'est d'appréhender la causalité du probable, si ce qui appelle explication, c'est la compréhension de l'adéquation des dispositions et d'une position, alors il faut accorder une égale attention à la vocation et au métier, il faut, pour reprendre le titre d'un ouvrage d'Hérault de Séchelles, ce constituant de 1793 parti à la recherche des lois de Minos, réfléchir à une théorie de l'ambition politique $^{71}$ et $s^{\prime}$ interroger, par exemple, sur le choix de la carrière de législateur de SaintJust qui, à vingt ans, écrit L'Esprit de la révolution, pendant républicain de L'Esprit des lois.

Dans cette perspective, la notion d'habitus de P. Bourdieu peut sans doute contribuer à faire avancer l'analyse, mais à condition d'adopter une définition large de la notion, comme totalité de l'histoire sociale intériorisée d'un sujet, comme incorporation du social au long d'une histoire de vie qui commence avec l'enfance et les interactions familiales ${ }^{72}$. On tiendra alors que ce système de dispositions socialement produites, sans former la totalité des déterminants des attitudes et des conduites (il faut compter avec la structure psychique ${ }^{73}$ ) les informent de manière décisive. En revanche, parce que l'habitus, dans sa conception classique, fait peu de cas de l'autonomie de l' "opérateur pratique" 74 qu'est l'individu, et plus largement de sa "compétence", c'est à dire de la propension au calcul de l'acteur social et de son travail d'auto-analyse et de stylisation, il n'est sans doute pas inutile, notamment dans l'univers politique, de souligner la dimension réflexive de la production de la personne. 


\section{Histoire et désir d'histoire}

Il faut confesser que toutes ces propositions, surtout les dernières, gardent un aspect hautement programmatique et que nous n'avons pas pu répondre aux interrogations que suscitaient certaines attitudes et certains comportements de Pierre Mendès France. On a pu montrer que de fortes contraintes ont pesé sur la production de soi de Mendès France à partir du moment où ses prétentions à l'exercice du pouvoir ont commencé à prendre consistance ${ }^{75}$. "Outsider", paria en opposition aux parvenus ${ }^{76}$ du système politique, doté de ressources rares et au départ peu monnayables dans l'arène parlementaire de la IV République, assuré de soutiens pour l'essentiel externes au Parlement, cette position dominée l'aurait conduit par force à un travail systématique de stylisation de soi et à une redéfinition de la politique et de son exercice, à miser, en quelque sorte, sur les "formes". Ce serait à ce moment que Mendès serait en quelque sorte devenu luimême. Marginalisé par G. Mollet puis exclu du jeu politique par de Gaulle et F. Mitterrand, privé, en dehors des intellectuels, de la plupart de ses alliés extérieurs au champ politique (notamment de la haute fonction publique), il n'a peut être eu d'autre choix que de rester fidele à lui même, à son identité de Cassandre, d'autre possibilité que de demeurer en dehors du jeu pour avoir quelque chance d'y revenir et d'apparaitre comme un recours possible en cas de crise, annoncée et attendue, du régime de la Vème République. Successeur et prédécesseur de de Gaulle, il semble qu'il prétendait lui succéder de nouveau?

Reste qu'une analyse des attributs et des ressources de Mendès France dit peu de ses prédispositions sociales. Faute d'indications que le biographé Mendès a tu à ses biographes, en dehors de l'autorité personnelle et du sentiment de sa particularité qu'il a tiré de ses succès précoces, les seules "propositions" concernent son surinvestissement intellectuel et sa volonté d'exemplarité qu'on peut associer à son parcours scolaire et politique et peut être à sa judéité : être l'envers des représentations stigmatisantes de l'antisémitisme ${ }^{77}$.

Au total, et pour conclure ces réflexions trop éparses et encore une fois largement hypothétiques, on souhaiterait réincarner les études du personnel politique, redonner chair à l'analyse du jeu politique. On suggère que l'étude du personnel politique devrait, parallèlement à l'analyse des mécanismes d'apprentissage et de formation, s'orienter vers l'émergence des "vocations" et des identités subjectives et objectives. On convient que ces propositions alourdissent l'analyse, qu'il s'agit là d'une machinerie complexe à manier, faite de dispositions, du travail, plus ou moins conscient, de construction et de présentation de soi, et de jeu de l'acteur par rapport à des objectivations sociales, mais elle nous parait indispensable. 


\section{Pôle Sud : biographies et politique}

1. Kafka, Le process.

\section{Notes}

2. M. Ozouf, Le Panthéon, in P. Nora, Les lieux de mémoire, La République, Paris, Gallimard, 1984. Dans le même ouvrage, A. Ben Amos, Les funérailles de Victor Hugo ; J. M Goulemot-E. Walter, Les centenaires de Voltaire et de Rousseau. Voir encore J C Bonnet, Les morts illustres, in P. Nora, Les lieux de mémoire, La Nation, vol 2, Paris, Gallimard, 1986.

3. L. Ferry, Les héros sont au chômage, L'Express, Avons-nous encore de grands hommes, 6-12 Juillet, 1990.

4. $P$. Bourdieu, Le mort saisit le vif, Actes de la recherche en sciences sociales, $n^{\circ} 32-33,1980$. M. Halbwachs, $L a$ mémoire collective, PUF, 1968.

5. A. Compagnon, La Troisieme République des Lettres, Le Seuil, 1983. B. Pudal, Nizan tel que reçu, Table Ronde "Intellectuels engagés d'une guerre à l'autre", préparatoire au colloque, "Les dictionnaires biographiques du mouvement ouvrier", CNRS, Paris, 1993.

6. J. P Rioux, Tombeau pour Mendès France, Vingtième Siècle, 1984, $\mathrm{n}^{\circ}$ 2, pp 43-54. C'est explicitement pour nettoyer la peinture de l'histoire mendésienne de repeints trop épais, pour débarrasser la perception de la réalité des "entrelacs du réel, du mémorisé et du commémoré" qui commençaient à l'obscurcir que l'Institut d'Histoire du Temps Présent décide de réunir en 1984 un colloque sur l'expérience gouvernementale de P. Mendès France et la postérité du mendésisme. J. P Rioux, A la recherche du mendésisme, in Institut d'Histoire du Temps Présent, Pierre Mendès France et le mendésisme, L'expérience gouvernementale (1954-1955) et sa postérité, Fayard, 1985, p 460.

7. A. Collovald, Identité (s) stratégiques (s), Actes de la Recherche en sciences sociales, ${ }^{\circ} 73,1988$.

8. F. G Bailey, Les règles du jeu politique, Paris, PUF, 1971. M. Dobry, Sociologie des crises politiques, Paris, FNSP, 1986.

9. Hegel, Leģons sur la philosophie de l'histoire; T. Carlyle, Les héros.

10. J. P Vernant, L'individu, la mort, l'amour, Gallimard,1989; N. Loraux, La "belle mort" spartiate, in Les expériences de Tirésias, Gallimard,1989.

11. C. Lefort, Mort de l'immortalité ? Le Temps de la Reflexion, 1982. O. Rank, Der Mythus von der Geburt des Helden, 1909. N Heinich, La gloire de Van Gogh, Les Editions de Minuit, 1991.

12. M. Fumaroli, L'äge de l'éloquence. Rhétorique et "res literaria" de la Renaisance à l'époque clasique, Genève, Droz, 1980.

13. Voltaire, in M. Ozouf, art cit.

14. Qui, par exemple, font défaut à de Gaulle en 1945. L'autorité acquise dans une période de suspension des règles s'accommode difficilement d'un retour à l'ordre, situation qui tient moins à la personnalité du "grand homme" qu'à l'évolution du rapport de forces politique tel qu'il s'inscrit d'abord sur la scène de la représentation.

15. M. Weber, Economie et Societé, Paris, Plon, 1971, p 475 sq.

16. R. Goetz, in O. Rudelle, P. Mendès France et l'identité républicaine, in IHTP, op cit, p 479.

17. Et plus largement par les historiens de la IVème et de la Vème République. Ce texte était largement écrit au moment de la soutenance de thèse de B. Gaïti, De la quatrième à la cinquieme République, Les conditions de la réalisation d'une prophétie. Paris, 1992.

18. A cet égard, il suffira de relever la solennité des titres des derniers colloques consacrés à P. Mendès France, "Pierre Mendès France et l'économie ; pensée et action" (IHTP, 1989); "Pierre Mendès France et la morale en politique", (Grenoble, 1990) ; "Pierre Mendès France et le rôle de la France dans le monde" (Paris, 1991). Dans son Mendes France ou la renovation politique (FNSP, 1993), J. L Rizzo, commence sur un hommage de R. G Schwartzenberg, et poursuit en indiquant que la biographie de J. Lacouture lui apparaît pour l'instant définitive, p 14. A contrario, voir notamment J. F Sirinelli et M. Sadoun, IHTP, op cit. 


\section{Histoire et désir d'histoire}

19. L. Boltanski, L. Thévenot, De la justification, les économies de la grandeur, Paris, Gallimard, 1991.

20. Idem.

21. Il ne faudrait pas croire avoir réglé définitivement le problème du rapport aux valeurs en appréhendant la seule version "démocratique" de la cité civique. Car les analyses néo-wébérienne ou néo-schumpéterienne de la spécialisation et de la professionnalisation de la politique sont tout autant concernées dans la mesure où elles risquent en permanence de servir de caution scientifique aux humeurs anti-institutionnelles de l'époque ou à un populisme facile. A moins encore qu'elles ne succombent à leur tour, dans une conjoncture actuelle de deuil des idéologies, et comme prises de paresse ou de remords, à une idéalisation des incarnations les plus légitimes de la démocratie parlementaire. C'est dire que le chercheur est pris sous le charme de la cité démocratique, quelque soit l'endroit d'où il la regarde.

22. P. Mendès France; intervention radiophonique du 24 novembre 1944, In A. J Tudesq, Pierre Mendès France et la radiodiffusion, in IHTP, op cit, p 207.

23. Selon les termes de F. Giroud; voir l'interrogation de P. Mendès France : “Est-ce L'Express qui m'a donné l'impulsion décisive ou ma première tentative de former le gouvernement, en juin 1953, qui lui a donné son premier élan.", in J. Lacouture, P. Mendes France, op. cit, p 204. Coll, L'Express, L'aventure du vrai, Albin Michel, 1979. S. Siritsky, F. Roth, Le roman de L'Express, Ateliers M. Jullian,1980. E. Tourniaire, L'Express et le phénomène mendésiste (1953-1956), in IHTP, pp 195-204. Cette interrogation sur L'Express pourrait sans difficulté être transposée au Nouvel Observateur des années 60.

24. P. Viasson-Ponté, Lettre ouverte aux hommes politiques, Albin Michel, 1976.

25. P. Viansson-Ponté, op cit, et J. Almaric, Une certaine idée de la politique, in Le Monde, 20 Octobre 1982.

26. J. Lacouture, op cit, p 206.

27. P. Rotman, La diaspora mendesiste, Pouvoirs, $\mathrm{n}^{\circ} 27,1983$.

28. M. Mauss, Essais de sociologie, Seuil, 1971.

29. Le Monde, 20 Octobre1982.

30. L'Heure de Vérité, Antenne 2, 3/12/1984. Et, pour L. Fabius, Le Monde, 22 Août 1991.

31. G. Suffert, Les Cabiers de la République, 1960, in J. P Rioux, IHTP, 470.

32. M. Rocard, Le Nouvel Observateur, 23/10/1982.

33. L. Pinto, L'intelligence en action : Le Nouvel Observateur, A. M Métailié, 1984. Pour cette forme de journalisme qui s'exprime à la première personne, s'instaure, par le biais de la commémoration, un mécanisme de légitimation en boucle.

34. "Celui qui s'occupe de ce dont il ne devrait pas" selon J P Sartre ou selon Brunetière, "Ceux qui raisonnent avec autorité sur des choses de leur incompétence", à propos des "intellectuels pétitionnaires" de l'Affaire Dreyfus. En d'autres termes, "I 'intellectuel à la française consacre le droit des spécialistes de l'universel à intervenir et à prendre parti sur le marché des biens politiques. Pour une approche voisine, P. Ory, Dernieres questions aux intellectuels, Olivier Orban, 1990.

35. J. F Sirinelli, Les intellectuels et $P$. Mendès France : un phénomène de génération ?, in IHTP, p 88. P. Godt : "Non conformiste dans un monde politique de médiocrité orthodoxe", Mendès France et les gauches, 1953-1958, Pouvoirs, op cit, p.69. Sur cette veine intellectualocentriste, voir B. Gaïti op cit ; A. Collovald : Histoire d'un mot de passe, le poujadisme, Geneses, 3, 1991. Sur le capital scolaire des parlementaires de la IVème République, D. Marwick, Political decision-makers, Glencoe, Free Press, 1961.

36. R. Aron, L'Opium des intellectuels, Calmann-Lévy, 1955. Sur les réserves de R. Aron à l'égard de Mendès, le ton de son éditorial dans L'Express du 22 octobre 1982. Surtout, ce jugement qui pose correctement le problème de la légitimité mendésienne : "Alors le mendésisme, c'est un mythe des intellectuels", Pouvoirs, $n^{\circ} 28,1984$, "Conversation avec R. Aron”, février 1983, par Y. Coudé du Foresto, p178. Voir également 


\section{Pôle Sud : biographies et politique}

Le spectateur engage, Julliard, 1984.

37. J. Verdès-Leroux, Au service du Parti, le Parti communiste, les intellectuels et la culture, (1944-1956), Fayard/Minuit, 1983.

38. S. de Beauvoir, in J. F Sirinelli, art cit, 91, note 21.

39. Ceci ne vaut ni pour Camus, ni pour M. Merleau-Ponty, cf J. F Sirinelli, art cit, et C. Nicolet, Pierre Mendès France et le métier de Cassandre, Julliard, 1959.

40. Les Temps Modernes, La Gauche, $\mathrm{n}^{\circ}$ spécial, 1955.

41. Voir le double parrainage Sartre-Mendès du premier numéro du Nouvel Observateur.

42. C. Nicolet, Mendès France, Le (s) mendésisme (s) et la tradition républicaine, Pouvoirs, op cit, et Le métier de Cassandre, op cit.

43. Voir C. Nicolet, op cit ; P. H Simon, préface à C. Nicolet, op cit ; J. M Domenach, Présentation à S.M. Lipset, L'homme et la politique, Paris, Le Seuil, 1962 ; P. Thibaud, La philosophie de Pierre Mendès France, in IHTP, p 45-51. Sur l'engagement des catholiques, of E. Fouilloux, Les catholiques mendésistes 19531956, in IHTP, pp 71-86, d'où nous tirons cet appel pour le Front Républicain paru dans Le Monde du 23/12/1955: "Les catholiques peuvent voter à gauche" dont les signatures devaient être adressées à J. Bourdin. Pour une analyse de ce point, M. Sadoun, De la méthode aux valeurs, Pouvoirs, n²7, 1983.

44. M. Sadoun note justement que Mendès avait renvoyé aux élites du savoir leur propre image, Le mendésisme contre Mendès France, in IHTP, p 498.

45. Bourgès-Maunoury à l'adresse d'I Marrou.

46. P. Mendès France, Gouverner, c'est choisir, Julliard, 1955. La science économique et l'action, UnescoJulliard, 1954.

47. M. Winock, La République se meurt, Paris, Le Seuil, 1978.

48. J. Monnet, Mémoires, 1978, 372.

49. F. Bloch. Lainé, Profession fonctionnaire, Seuil, 1976 ; également, son témoignage, P. Mendès France et les hauts fonctionnaires, in IHTP, pp 53-57 : "aux yeux de beaucoup de membres de la fonction publique, pour lesquels il a été une référence, un conseiller, un recours, une espérance hors-pair.", p 53.

50. F. Fourquet, Les comptes de la puissance, histoire de la comptabilité nationale et du plan, Paris, Encres, 1980.

51. M. Halbwachs, op cit.

52. B. Gaïti, op cit.

53. L. Boltanski, L'espace positionnel, multiplicité des positions institutionnelles et habitus de classe, Revue Française de sociologie, 1973, 1.

54. C. Ginsburg, Mythes, traces, emblemes, Flammarion, 1989 ; ainsi que Le fromage et les vers, Flammarion,

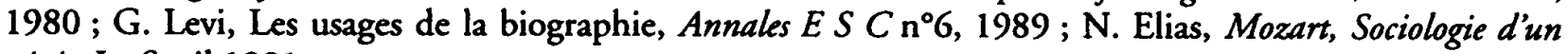
génie, Le Seuil,1991.

55. E. Auerbach, Mimésis, La representation de la réalité dans la littérature occidentale, Paris, 1968.

56. J. C. Passeron, Biogaphies, flux, itinéraires, trajectoires, Revue Fraņ̧aise de Sociologie, $\mathrm{n}^{\circ} 1,1990$; $\mathrm{P}$. Bourdieu, L'illusion biographique, Actes de la recherche en sciences sociales, n62/63,1986; J. Peneff, La méthode biographique, A Colin, 1990.

57. Pour un exemple de "prédestination", ce texte de J Lacouture sur P. Mendès France : "Né jacobin et laïque, élève modèle de l'école républicaine, brillant étudiant à la faculté de droit et des sciences politiques, "entré en radicalisme" à seize ans mais assez imbu de la multiple continuité de l'État pour admirer également Jaurès, Poincaré et Caillaux ; plus jeune avocat et plus jeune député de France ; secrétaire d'État à 


\section{Histoire et désir d'histoire}

trente et un ans, ministre à trente sept, tout en lui tend à l'exercice des responsabilités les plus hautes et les plus lourdes". J. Lacouture, P. Mendès France, Le Seuil, 1981.

58. Voir la présentation de ce numéro.

59. B. Lacroix, L'utopie communautaire, Paris, PUF, 1981 ; B. Pudal, Prendre parti, 1989, Paris, FNSP.

60. P. Braud, Le jardin des délices démocratiques, FNSP, 1991. P. Ansart, La gestion des passions politiques, L'Age d'Homme, 1983.

61. La remarque ne vaut pas la sociologie du comportement politique. Elle n'a pas grand sens non plus si on la rapporte à la sociologie ou à l'histoire, notamment à la perspective éliasienne qui allie socio-genèse et psycho-genèse.

62. De Gaulle, Mémoires d'Espoir, Le Livre de Poche, 1973, pp 39-40; cf également O. Guichard in P. Braud, op cit, p 190. “Je crois que si l'Histoire a un sens, elle n'en est pas moins, dans une certaine mesure, malléable... Si les hommes auxquels le pouvoir est confié interprètent convenablement la réalité historique à laquelle ils sont confrontés, ils peuvent favoriser des accouchements, les rendre moins pénibles, moins douloureux; ou au contraire freiner tel ou tel progrès." P. Mendès France, J. Lacouture, op cit.

63. de Gaulle, Le fil de l'épée, 10/18, 1962, (1ère édition Berger-Levrault, 1932) pp 53-54, et77.

64. R Merton, Eléments de théorie et de méthode sociologique, Plon, 1965, pp 140-164.

65. De Gaulle, Mémoires de Guerre, L'Appeh, Plon,1956, p 2.

66. N. Elias, La société des individus, Fayard,1991; La solitude des mourants, C. Bourgois,1987, p 49 sq.

67. L. Fabius, Le Monde, 22 Août 1991.

68. Dont il faudrait questionner le fonctionnement plus sérieusement qu'on l'a fait jusqu'à présent. Voir aussi la notion de "carrière", H. S Becker, Outsider, A M Métailié, 1985.

69. B. Lacroix, op cit.

70. Montesquieu, L'Esprit des Lois, Avertissement.

71. Hérault de Séchelles, Théorie de l'ambition, in Cahiers du Futur, 1974, $\mathrm{n}^{\circ} 2$.

72. P. Bourdieu, Reponses, Seuil, 1992, p103.

73. Au sens de Freud. S. Freud, W Bullitt, Le président T.W. Wilson, Payot, 1990. E. Erikson, Luther avant Luther, Flammarion, 1968 ; Gandi's Truth, New York, Norton,1969.

74. P. Bourdieu, Choses dites, Minuit, 1987, p 24.

75. B. Gaîti, "Les innovations mendésistes, avant même d'être liées à la promotion de ressources nouvelles sont un produit du déplacement des contraintes politique sur le candidat radical. Exclu du jeu collectif... il ne peut espérer apparemment de profits que de sa seule singularisation.", B. Gaïti, op cit, p 144.

76. Opposition qu' $\mathrm{H}$. Arendt applique aux juifs de l'Allemagne de Weimar.

77. A la suite de M. Robert, Roman des origines et origines du roman, on pourrait également opposer Mendes, l'enfant trouvé, au "bâtard", C. de Gaulle. 